\title{
Analyse des facteurs de contingence de la comptabilisation des investissements immatériels : cas des entreprises cotées à la Bourse Régionale des Valeurs Mobilières d'Abidjan
}

\author{
Ossonon Ahou Claudine \\ UFR des Sciences Economiques et de Gestion \\ Université Félix Houphouët Boigny de Cocody - Abidjan - Côte d'Ivoire
}

\begin{abstract}
Résumé : Le propos de cette recherche est d'analyser les facteurs de contingence de la comptabilisation des investissements immatériels. A partir d'un échantillon de 23 entreprises non financières cotées sur la BRVM, notre analyse de régression dénote que les entreprises activent les investissements immatériels pour se libérer des contraintes financières imposées par les contrats d'endettement. D'ailleurs, les grandes entreprises sont enclines à activer les investissements immatériels afin d'informer le marché financier sur la qualité de leurs projets contrairement à l'hypothèse des coûts politiques émise par la théorie politico-contractuelle de Watts et Zimmerman (1986).
\end{abstract}

Mots-clés : Système Comptable ; Immatériels ; Contraintes financières ; Contrat d'endettement ; Coûts politiques.

Digital Object Identifier (DOI): https://doi.org/10.52502/ijesm.v1i3.210 


\section{Introduction}

Depuis plusieurs décennies, les entreprises sont confrontées à un changement dans le processus de création de richesse. Les investissements immatériels que sont les brevets, les logiciels, les frais de recherche et développement... sont devenus les catalyseurs de la création de richesse des entreprises. C'est à juste titre que Gonne et Feudjo (2017) soutiennent que les immatériels sont les éléments autour desquels les entreprises bâtissent leurs stratégies de création de valeur et de développement.

Les normalisateurs comptables, conscients de cette nouvelle donne de l'économie, continuent l'harmonisation des normes comptables. Selon Thibierge (2001), en dépit d'une certaine convergence des instances de normalisation comptables, le traitement des dépenses immatérielles offre plusieurs options de comptabilisation dont peuvent profiter les entreprises relativement à leurs politiques comptables.

En vertu de l'intérêt que revêt la problématique de la comptabilisation des immatériels, la littérature théorique et empirique a mis en évidence des raisons susceptibles d'inciter les dirigeants d'entreprise à capitaliser les dépenses immatérielles : informer le marché financier pour une valorisation boursière (Bartov et Bodnar, 1996 ; Aboody et Lev, 1998), informer les créanciers pour se libérer des contraintes d'endettement (Daley et Vigeland, 1983 ; Watts et Zimmerman, 1986 ;Skinner, 1993 ;Thibierge, 1997, 2001 ; Dhaliwal et al, 1999 ; Landry et Callimaci, 2003, Cazavan-Jeny et Jeanjean, 2006, Charfi, 2006, Koumagnanou, 2018), minimiser les coûts politiques (Olivier, 2010; Mard et Marsat, 2011)...Cependant, la revue de la littérature nous a permis de constater que les conclusions des études américaines sont remises en cause par des recherches portant sur la décision d'activation des dépenses immatérielles effectuées dans des contextes autres que le contexte américain (français, allemand, européen).

Par ailleurs, selon le Système Comptable pour l'Harmonisation en Afrique du Droit des Affaires comme tout modèle comptable euro-continental, s'agissant de la comptabilisation des frais de recherche et de développement, « seules les dépenses relatives à des projets qui ont de sérieuses chances de réalisation technique et de rentabilité commerciale en raison de l'existence d'un marché potentiel peuvent être inscrites à l'actif $d u$ bilan ». En outre, ces projets doivent être nettement individualisés et leur coût distinctement établi pour être répartis dans le temps. Cette norme comptable, nonobstant sa révision, offre un traitement alternatif aux entreprises dans la comptabilisation des investissements immatériels (passage en charges versus inscription à l'actif) en fonction de leurs choix comptables.

Par conséquent, cette disposition comptable donnant la latitude aux dirigeants d'entreprise d'opérer des choix comptables en matière de comptabilisation des immatériels, il paraît judicieux d'analyser les motivations des dirigeants d'entreprises soumises au Système Comptable pour l'Harmonisation en Afrique du Droit des Affaires dans leurs politiques comptables. De cet état de fait, découle la question 
principale suivante: quels sont les facteurs explicatifs de la comptabilisation des investissements immatériels?

Ainsi, nous formulons les questions secondaires suivantes : les entreprises capitalisent - elles les immatériels pour informer le marché financier? Les entreprises activent-elles les immatériels pour se libérer des contraintes d'endettement? Les entreprises de grande taille passent-elles les immatériels en charge pour minimiser les coûts politiques ?

L'objectif principal de cette étude est d'analyser les facteurs de contingence de la comptabilisation des investissements immatériels. Il s'agira spécifiquement de :

-examiner la relation entre l'activation des investissements immatériels et la valorisation boursière ; -analyser le lien entre la capitalisation des investissements immatériels et l'endettement ;

-vérifier la relation entre le niveau d'activation des investissements immatériels et la taille.

Afin de répondre à notre problématique, nous testerons empiriquement sur un échantillon d'entreprises cotées à la Bourse Régionale des Valeurs Mobilières d'Abidjan (BRVM), des hypothèses empruntées à la théorie positive de la comptabilité et à la théorie du signal à partir d'une régression linéaire et d'une régression logistique.

D'ailleurs, cette recherche apporte deux contributions majeures. An plan théorique, cette étude contribue au débat relatif aux facteurs déterminants de la politique comptable des dirigeants d'entreprise en matière de comptabilisation des dépenses immatérielles. Au plan social, la présente recherche pourrait permettre au normalisateur d'une part, de cerner les comportements et les stratégies de communication des entreprises cotées à la BRVM en matière de comptabilisation des investissements immatériels et d'autre part, combler les insuffisances du système comptable de l'Organisation pour l'Harmonisation en Afrique du Droit des Affaires (Syscohada) afin d'éviter la manipulation des résultats comptables. Pour les investisseurs, ladite recherche pourrait leur permettre d'apprécier les stratégies de communication financière des entreprises afin d'orienter leurs décisions d'investissement sur le marché financier régional.

Pour cerner les contours de cette recherche, nous la subdiviserons en trois sections. D'abord, la première section sera réservée à la revue de littérature et à la formulation des hypothèses de recherche. Ensuite, la seconde section s'évertuera à exposer la méthodologie de l'étude. Enfin, la troisième section sera consacrée à l'interprétation et à la discussion des résultats.

\section{Revue de littérature et hypothèses de recherche}

\subsection{Théories explicatives de la comptabilisation des investissements immatériels}

La notion d'investissement immatériel ne fait pas l'objet de définition admise donc universelle. Il n'y a pas de consensus sur une définition précise et unique (Cahn, 2012). En effet, en l'absence de références 
normatives claires, la conceptualisation n'a aucunement abouti à une définition consensuelle (Alcouffe et Louzzani, 2003). Toutefois, dans le cadre de notre étude, nous retenons la définition de Martory et Pierrat (1996) qui définissent les investissements immatériels comme des dépenses immatérielles engagées aujourd'hui dans l'espoir de revenus futurs directs ou indirects.

En dépit des efforts de normalisation, les règles de comptabilisation des immatériels ont dû mal à reconnaître les immatériels comme étant tous des actifs générateurs de revenus (Nils et al, 2000). Ce trou noir dans la comptabilisation des immatériels s'explique par leur caractère protéiforme d'une part, et par la difficulté à évaluer leur contribution à la création de valeur des entreprises. Par conséquent, l'existence du traitement alternatif de la comptabilisation des investissements immatériels ont amené les chercheurs à s'interroger sur les motivations des entreprises relatives à leur décision d'activation des immatériels. Cette préoccupation des chercheurs a fait émerger des théories explicatives du comportement des dirigeants d'entreprise en matière de comptabilisation des immatériels. Il s'agit, entre autres, de la théorie positive de la comptabilité de Watts et Zimmerman (1986), de la théorie de l'agence (1976) et de la théorie du signal (Ross, 1977).

La théorie de l'agence de Jensen et Meckling (1976) postule que l'entreprise est un entrelacement de conventions entre des acteurs rationnels guidés par la maximisation de leurs propres utilités. Cette théorie énonce l'existence de conflits d'intérêt entre les actionnaires et les créanciers. Pour réduire ces conflits d'intérêt, les entreprises rédigent des clauses liées au contrat d'endettement (debts covenants). Au regard de ces debt convenants, les entreprises font des choix comptables pour maximiser les résultats comptables afin d'éviter la prise de contrôle par les créanciers ou la renégociation des clauses contractuelles avec les institutions de crédit (Thibierge, 2001).

La théorie de la réglementation initiée par Posner (1974) considère le processus politique comme une compétition entre les acteurs pour maximiser leurs intérêts. Cette théorie soutient que le but des réglementations est d'effectuer des transferts de richesse, les nombres comptables, précisément, le résultat comptable et les capitaux propres.

Les entreprises exposées à des pressions politiques sont incitées à gérer leurs résultats à la baisse afin de minimiser les coûts politiques. Pour ce faire, les dirigeants passent en charges les dépenses immatérielles afin de réduire le résultat fiscal.

Inspirée de la théorie de l'agence et de la théorie de la réglementation, cette théorie initiée par Watts et Zimmerman (1986) vise à prédire et à expliquer les pratiques comptables des entreprises. Cette théorie est qualifiée de théorie politico-contractuelle de la comptabilité par Raffournier (1990). Ces auteurs ont modélisé le comportement des acteurs face aux choix comptables au travers de trois hypothèses: l'endettement, la taille et la rémunération des dirigeants. Ces auteurs ont mis en exergue les motivations de la gestion des résultats. Selon Watts et Zimmerman (1978), les entreprises sont soumises à un processus politique, source de contraintes politiques. Ceux-ci définissent le processus politique comme 
une lutte entre des personnes qui cherchent à maximiser leurs intérêts personnels. De ce fait, les hommes politiques, contraints de distribuer la richesse nationale, imposent aux entreprises le montant d'impôt à payer en fonction de leur taille. Pour éviter des coûts politiques, ces entreprises ont intérêt à minimiser leurs résultats. Ainsi, les grandes entreprises devraient privilégier les méthodes comptables minorant le résultat.

Fondée sur l'asymétrie informationnelle entre l'entreprise et ses stakeholders, la théorie des signaux (Ross, 1977) postule que les dirigeants d'une entreprise possédant une information meilleure à celle de ses pourvoyeurs de fonds, doivent mettre en place une stratégie de communication efficace pour convaincre les investisseurs. Pour ce faire, ils ont recours au signal, décision financière porteuse de conséquences financièrement négatives pour son initiateur au cas où ce signal se révélerait erroné. A contrario, un signal positif émis par les dirigeants d'une société, peut permettre d'anticiper de meilleures performances futures et engendrer une augmentation du cours de l'action de la valeur de la société.

Par ailleurs, la comptabilisation des immatériels accroît les problèmes d'agence entre l'entreprise et ses parties prenantes (Draief, 2006). Conscients de cet état de fait, les dirigeants d'entreprise sont très enclins à activer les dépenses immatérielles pour informer le marché financier sur la qualité de leurs projets, d'où sur leurs performances futures. Ce qui contribuerait à augmenter la valeur informative des données comptables aux yeux des investisseurs sur le marché financier.

\subsection{Etudes empiriques portant sur les facteurs de contingence de la comptabilisation des immatériels et formulation des hypothèses de recherche}

Les théories explicatives de la comptabilisation des investissements immatériels ont fait l'objet de débats empiriques controversés dans les contextes américain, canadien, européen, français, allemand et africain. En effet, Skinner (1983), à partir de 504 entreprises américaines observées sur la période de 1985 à 1987, fournit des preuves sur la relation transversale entre les opportunités d'investissement des entreprises, leurs contrats de dette, leur taille et leurs choix de procédures comptables. Toujours dans le contexte américain, comme Skinner (1983), sur la base de 313 entreprises américaines, Daley et Vigeland (1983) examinent le lien entre la comptabilisation des dépenses de R\&D avant 1974 et l'endettement ainsi que la taille des entreprises. L'analyse témoigne que les entreprises ayant capitalisé les coûts de R\&D étaient plus fortement endettées et étaient de petite taille que celles qui ont passé en charges les coûts de R\&D.

Dans la même veine, Dhaliwal et al (1999) examinent les effets de l'ensemble des opportunités d'investissement sur la décision de capitaliser les coûts de recherche et développement avant 1974. Leur étude révèle que les entreprises à forte croissance sont plus enclines à capitaliser les frais de recherche et développement pour augmenter leurs résultats. Ces auteurs soutiennent également que la taille de l'entreprise et les contrats de dette expliquent aussi la décision de capitaliser les coûts de recherche et 
de développement. Par ailleurs, les résultats obtenus par Dhaliwal et al (1999) sont similaires à ceux de Defond et Jiambalvo (1994) en observant un échantillon de 94 entreprises, plus précisément concernant les contrats de dette. Bartov et Bodnar (1996), observant un échantillon de 788 entreprises américaines, montrent que les entreprises souhaitant accroître leur valeur de marché, sont incitées à réduire l'asymétrie informationnelle en opérant des choix comptables qui rendent les états financiers plus informatifs pour les investisseurs, après avoir contrôlé la taille, l'endettement et les frais financiers des entreprises.

Après 1974, en raison de l'absence du traitement alternatif en matière de comptabilisation des frais de $R \& D$, des études se sont limitées à tester la valorisation par le marché financier des dépenses de $R \& D$ (Thibierge, 1997). Suivant ce principe, Aboody et Lev (1998) analysent la pertinence de la capitalisation des frais de $R \& D$ sur une période de 10 ans. Leur étude révèle que les dépenses en $R \& D$ sont positivement associées aux variables de marché (Cours et rendement des actions).

Dans le contexte canadien, les travaux de Landry et Callimaci (2003) indiquent que la probabilité de capitaliser les dépenses de R\&D augmente pour les entreprises qui sont plus endettées et dont les flux de trésorerie d'exploitation sont plus élevés. Cependant, la probabilité de capitaliser les dépenses de $R \& D$ diminue pour les grandes entreprises, les entreprises à propriété plus concentrée et les entreprises très rentables.

Les motivations qui sous-tendent la comptabilisation des immatériels ont fait également l'objet de débats empiriques dans le contexte européen en raison du traitement alternatif en matière de comptabilisation des immatériels qu'offrent les systèmes comptables inspirés du modèle comptable continental. En effet, Thibierge (1997), dans le contexte européen, à partir d'un échantillon de 1016 entreprises européennes cotées et d'un échantillon de 843 entreprises non cotées aboutit aux résultats suivants : pour l'échantillon d'entreprises cotées de grande taille, aucune relation linéaire n'a été trouvée entre l'activation des immatériels et la valorisation par le marché financier; les entreprises de grande taille ont tendance à choisir des procédures comptables qui permettent de majorer les résultats, contrairement à l'hypothèse des coûts politiques; une relation négative entre l'endettement et l'activation des immatériels. Ces résultats sont en contradiction avec ceux obtenus dans le contexte américain et ceux trouvés avec l'échantillon d'entreprises non cotées et de petite taille.

Comme Aboody et Lev (1998), Cazavan-Jeny et Jeanjean (2006) examinent la pertinence de la capitalisation des frais de Recherche et Développement (R\&D) pour le marché financier français. A partir d'un échantillon de 197 entreprises cotées observées sur la période de 1993 à 2002, l'analyse révèle que la capitalisation des frais de R\&D est associée négativement au prix des actions ainsi qu'au rendement boursier, contrairement aux résultats d'Aboody et Lev (1998) trouvés dans le contexte américain. Il va s'en dire que la capitalisation des dépenses en R\&D n'est pas pertinente pour les investisseurs sur le marché financier français. Dans la même optique, Charfi (2006) examine les facteurs 
explicatifs de l'activation des dépenses de R\&D dans le contexte français. A travers un modèle logit appliqué à un échantillon de 253 entreprises françaises non cotées observées sur la période 2000 à 2002, ces auteurs concluent que les entreprises qui capitalisent leurs dépenses de $R \& D$ sont de petite taille et plus endettées et moins performantes que celles qui passent leurs dépenses en charges.

Une étude similaire a été réalisée sur un échantillon de 586 entreprises allemandes par Eierle et Wencki ((2016). Les résultats indiquent que les entreprises sont motivées à capitaliser les frais de développement pour se libérer des contraintes d'endettement. En outre, l'étude dénote que les entreprises optent pour la capitalisation des frais de développement de façon opportuniste et pour informer les utilisateurs des états financiers sur leurs performances globales.

En Afrique, les systèmes comptables en vigueur dérivent du modèle comptable continental qui est caractérisé par une connexion étroite entre la comptabilité et la fiscalité. Ainsi, en raison de l'existence du traitement alternatif en matière de comptabilisation des dépenses en $R \& D$, des réflexions ont été menées pour déceler les raisons qui sous-tendent les choix comptables des entreprises. Précisément, Turki et al (2006) examinent les facteurs explicatifs de l'activation des immatériels. Sur la base d'une régression linéaire et logistique portant sur échantillon de 50 entreprises tunisiennes observées en 2003, l'analyse dénote que les entreprises décident d'activer les dépenses immatérielles pour réduire les contraintes d'endettement. Toutefois, l'hypothèse de réduction des coûts politiques a été infirmée. Dans le même contexte, Chabchoub et Bouri (2011) analysent un échantillon de 21 entreprises tunisiennes cotées et 51 entreprises non cotées sur la période de 2002 à 2005. Ces chercheurs prouvent que les facteurs déterminant le choix d'activation des investissements immatériels sont les contraintes d'endettement comme Turki et al (2006) ainsi que la taille pour les entreprises non cotées. Pour ces chercheurs, la qualité d'audit est aussi un facteur motivant les choix comptables pour les entreprises cotées. L'Afrique de l'ouest n'est pas restée en marge de cette recherche. Koumagnanou (2018), à l'aide d'un guide d'entretien et d'un questionnaire administré à 37 entreprises togolaises, soutient que les entreprises comptabilisent les immatériels au bilan pour se libérer des contraintes financières imposées par les contrats d'endettement, conformément aux prédictions de la théorie positive de la comptabilité. D'ailleurs, cet auteur trouve également que les entreprises font des choix comptables non seulement pour minimiser l'impôt, mais aussi pour augmenter la richesse des actionnaires.

Le tour d'horizon relatif à la littérature liée aux déterminants de l'activation des immatériels fait ressortir deux constats :

-les résultats portant sur ce type études sont mitigés d'un contexte à un autre ;

-à notre connaissance, dans le contexte ouest africain, une seule étude (Koumagnanou, 2018) a été menée ; 
Au regard de ces constats, il paraît opportun de mettre en évidence les facteurs discriminant la politique comptable en matière de comptabilisation des immatériels des entreprises opérant dans le contexte ouest africain.

Pour répondre à cette problématique, nous formulons les hypothèses à tester.

Conformément à la théorie du signal, aux travaux de Bartov et Bodnar (1996) et à ceux d'Aboody et Lev (1998), nous soutenons que :

$\mathrm{H}_{1}$-les entreprises activent les investissements immatériels pour informer les investisseurs sur la qualité de leurs projets.

Nous prédisons un lien positif entre les investissements immatériels et la valorisation boursière.

Suivant les prescriptions de la théorie positive de la comptabilité et les études de Skinner (1993) et selon les travaux de Daley et Vigeland (1983), nous postulons que :

$\mathrm{H}_{2}$ - les entreprises capitalisent les investissements immatériels pour se libérer des contraintes financières imposées par les contrats d'endettement ;

Comme Thibierge (2001), nous formulons 2 sous-hypothèses :

$\mathrm{H}_{2-1}$ - les entreprises plus endettées activent les investissements immatériels pour augmenter leurs résultats ;

$\mathrm{H}_{2-2}$ - les entreprises activant les immatériels ont des ratios de couverture des frais financiers plus faibles ;

$\mathrm{H}_{3}$ - les entreprises de grande taille passent en charge les investissements immatériels.

Nous testerons cette troisième hypothèse en considérant la taille comme variable de contrôle.

\section{Méthodologie de l'étude}

\subsection{Sélection de l'échantillon et données de l'étude}

L'étude empirique est axée sur un échantillon initial de 45 entreprises cotées à la BRVM. De cet échantillon, sont exclues les entreprises financières (banques, assurances et sociétés de crédit-bail) en raison de leurs spécificités économiques et financières. Sont retirées de l'échantillon, les entreprises présentant des données incomplètes. Après apurement de la base, 23 entreprises ont été retenues comme constituant l'échantillon final. Cet échantillon est observé sur une période de 7 ans allant de 2012 à 2018. Il est réparti selon les secteurs d'activité que sont les secteurs Distribution, Industrie, Agriculture, Transport et Secteurs publics dans le tableau ${ }^{\circ} 1$. 
Tableau n ${ }^{\circ} 1$ - Répartition sectorielle des entreprises de l'échantillon

\begin{tabular}{|l|l|c|}
\hline Secteur d'activité & Effectif & Proportion \\
\hline Distribution & 5 & $22 \%$ \\
\hline Industrie & 11 & $48 \%$ \\
\hline Agriculture & 2 & $9 \%$ \\
\hline Transport & 1 & $4 \%$ \\
\hline Secteurs publics & 4 & $17 \%$ \\
\hline TOTAL & 23 & $100 \%$ \\
\hline
\end{tabular}

\section{Source : Auteur}

Pour tester nos hypothèses, nous avons collecté des données comptables au travers des états financiers publiés sur le site de la BRVM et des données boursières à partir des bulletins officiels de la cote publiés également sur le site web de la BRVM.

\subsection{Mesures des variables de l'étude}

Concernant la mesure de l'investissement immatériel, logiquement, les investissements immatériels sont formées d'une part par les dépenses immatérielles inscrites en charges (dépenses en publicité et frais de formation du personnel) et d'autre part, par les dépenses immatérielles inscrites à l'actif du bilan qui sont capitalisées, appelées immobilisations incorporelles (frais de recherche et développement, logiciels, brevets, droit au bail ...). Toutefois, le tour de la littérature empirique relative aux immatériels nous a permis de constater que très souvent les auteurs (Thibierge, 1997, 2001 ; Charfi, 2006 ; Turki et al, 2006 ; Chabchoub et Bouri, 2011) mesurent les immatériels seulement par la partie inscrite à l'actif du bilan, c'est-à-dire par le poste immobilisation incorporelles, à l'exception des travaux de Cazavan-Jeny et Jeanjean (2006) ainsi que ceux de Saoussen (2010). La principale raison de ce choix réside dans le fait que les données sur les immatériels inscrits en charge sont indisponibles car agrégées dans les charges d'exploitation. En outre, dans le contexte européen, seuls les montants des immatériels activés sont repérables dans les états financiers publiés.

Par conséquent, similairement aux études de Thibierge $(1997$; 2001) et Charfi (2006) réalisées dans le contexte français, nous retenons dans le cadre de notre recherche comme mesure des investissements immatériels, le montant des immobilisations incorporelles qui est la résultante des choix discrétionnaires des dirigeants (Thibierge, 1997 ; 2001). Comme Thibierge (1997), Chabchoub et Bouri (2011) le montant des immobilisations incorporelles sera normé par le total de l'actif Comptable : INC/AC = Immobilisations incorporelles nettes / Actif Comptable. 
La théorie du signal stipule qu'en raison de l'asymétrie informationnelle entre les dirigeants et les actionnaires, ceux-ci publient l'information sur le marché financier pour émettre un signal sur les performances futures de l'entreprise. Spécifiquement, les investissements immatériels dans l'imaginaire collectif, revêtent un caractère secret et confidentiel (Thibierge, 1997). Pour ce faire, les entreprises publient l'information relative à l'immatériel sur le marché financier pour émettre un signal à l'endroit des investisseurs. Si ce signal est perçu positivement par ces derniers, l'entreprise émettrice de ce signal sera valorisée positivement. Dans le cas contraire, l'entreprise sera valorisée négativement par le marché financier. D'ailleurs, Amir et Lev (1996) soutiennent que la capitalisation des dépenses en Recherche et Développement est une information donnée aux partenaires pour les renseigner sur la qualité de l'activité de l'entreprise et ses perspectives de croissance. C'est ainsi que dans les études empiriques, les auteurs utilisent ordinairement le Q de Tobin pour mesurer la valorisation du capital immatériel par le marché financier. En effet, un Q de Tobin supérieur à l'unité signifie qu'il y a présence d'une source de valeur non prise en compte par la comptabilité générée par le capital immatériel (Bharadwaj et al, 1999; Gerpott, 2008). Cet indicateur est également le signe qu'une entreprise a des opportunités d'investissement rentables et inversement.

Toutefois, en raison de l'indisponibilité des données pour la mesure du Q de Tobin théorique, le Q de Tobin ajusté est utilisé dans la littérature empirique comme proxy de la mesure théorique du Q de Tobin (Thibierge, 1997, 2001 ; Bharadwaj et al, 1999 ; Turki et al, 2006 ; Bellalah et al, 2010 ; Chabchoub et Bouri, 2011 ; Maaloul et Zhégal, 2015 ; Gonne et Feudjo, 2017). Effectivement, à partir d'une série de régression, Chung et Pruitt (1994) prouvent qu'au moins 96,6\% de la variabilité du Q de Tobin est expliquée par le Q de Tobin ajusté. Dans le cadre de notre recherche, comme Thibierge (1997, 2001), nous retenons le Q de Tobin ajusté pour mesurer la valorisation du capital immatériel par le marché financier : $\mathrm{Q}$ de Tobin $=($ Valeur de marché des capitaux propres + Valeur comptable des dettes $) /$ Actif comptable

Dans le tableau $\mathrm{n}^{\circ} 2$, nous nous attèlerons à décrire les variables explicatives retenues pour tester nos hypothèses de recherche inhérentes aux facteurs illustratifs de la comptabilisation des investissements immatériels. La littérature théorique et empirique identifie plusieurs facteurs, entre autres, l'endettement, la taille... En plus de ces variables, nous intégrerons dans le modèle explicatif des variables de contrôle telles que la capacité d'autofinancement global et la capacité d'autofinancement d'exploitation. Ces variables sont résumées dans le tableau n². 
Tableau n ${ }^{\circ} 2$ : Synthèse des variables explicatives

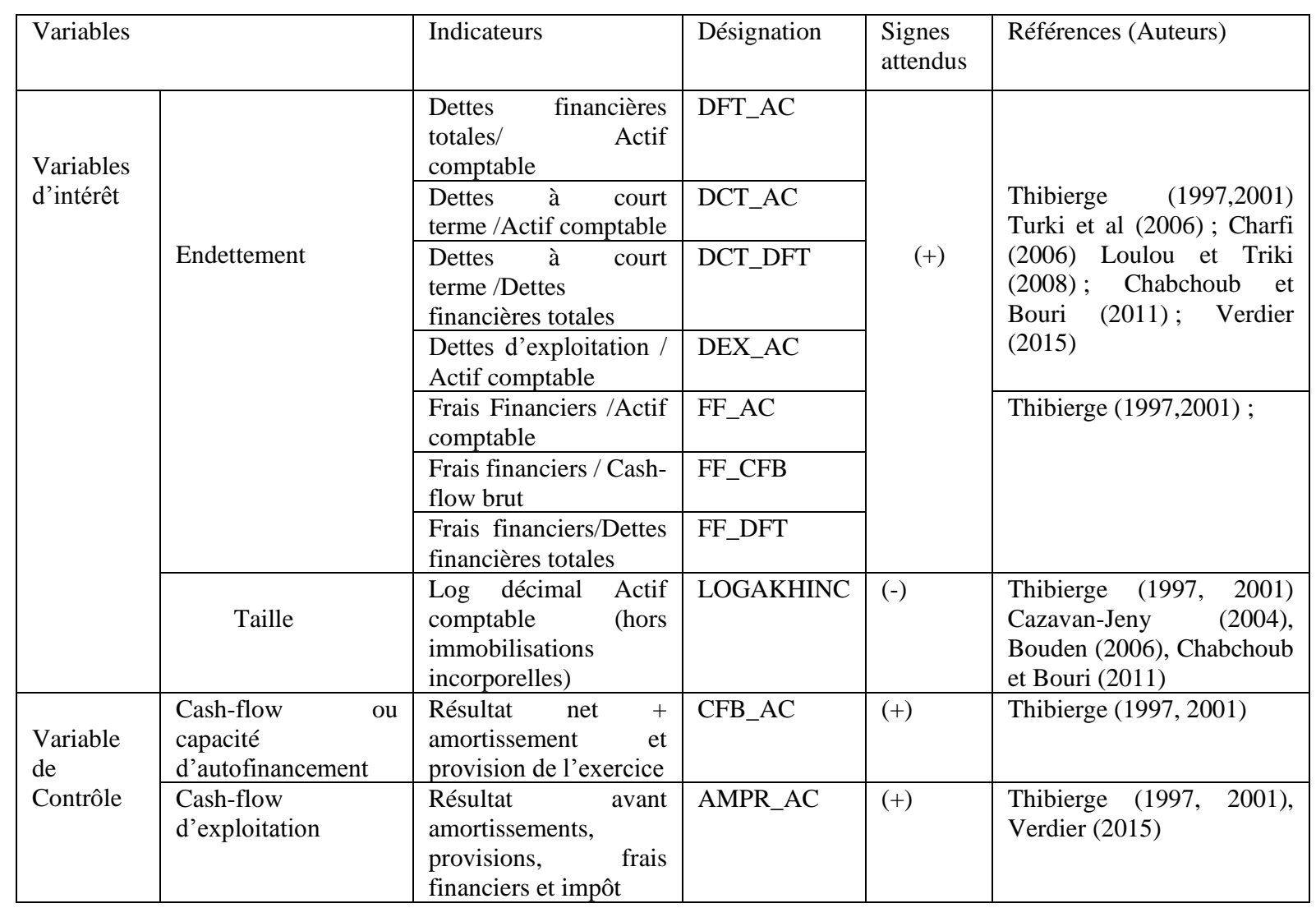

\section{Source : Auteur}

\subsection{Modèles de l'étude et méthodes d'analyse}

Pour répondre à notre objectif de recherche, nous proposons de tester empiriquement deux modèles de régression. Premièrement, conformément aux travaux de Thibierge (1997, 2001), nous testons l'hypothèse $\mathrm{H}_{1}$ par la vérification de la relation entre le montant des immatériels activés et le $\mathrm{Q}$ de Tobin, comme indicateur de valorisation des immatériels par le marché financier. Dans ce premier modèle, nous inclurons la taille comme variable de contrôle. D'ailleurs, cette régression permettra d'appréhender la pertinence de l'activation des investissements immatériels pour les investisseurs sur le marché financier régional. Deuxièmement, Comme Saada (1995), Thibierge (1997, 2001) et Loulou et Triki (2006), nous procéderons par une régression logistique pour examiner le pouvoir explicatif des variables financières retenues dans la comptabilisation des investissements immatériels afin de tester les hypothèses $\mathrm{H}_{2}$ et $\mathrm{H}_{3}$. Ce deuxième modèle utilise comme variable dépendante, une variable dichotomique $\mathrm{Y}$ qui prend la valeur 1 pour les entreprises qui activent les investissements immatériels et 0 pour celles qui les passent en charges. Les modèles à estimer, inspirés des travaux de Thibierge (2001) et Turki et al (2006) sont :

Modèle $1:$ INC_AC $=\alpha_{0}+\alpha_{1} \mathrm{Q}_{\mathrm{i}, \mathrm{t}}+\alpha_{2}$ LOG_AKHINC $_{\mathrm{i}, \mathrm{t}}+\varepsilon_{\mathrm{i}, \mathrm{t} .}$ 
Avec :

INC_AC : proportion des actifs incorporels dans le total actif de l'entreprise i à l'année t $\mathrm{Q}: \mathrm{Q}$ de Tobin de l'entreprise i à l'année $\mathrm{t}$

LOG_AKHINC: taille de l'entreprise i à l'année t

Modèle 2:Y $=\alpha_{0}+\alpha_{1}$ DFT_AC $\mathrm{i}_{\mathrm{i}, \mathrm{t}}+\alpha_{2} \mathrm{DCT} \_\mathrm{AC} \mathrm{C}_{\mathrm{i}, \mathrm{t}}+\alpha_{3} \mathrm{DCT} \_\mathrm{DFT} \mathrm{i}_{\mathrm{i}, \mathrm{t}}+\alpha_{4} \mathrm{DEX} \_\mathrm{AC} \mathrm{C}_{\mathrm{i}, \mathrm{t}}+\alpha_{5} \mathrm{FF}_{-} \mathrm{AC} \mathrm{C}_{\mathrm{i}, \mathrm{t}}+$ $\alpha_{6} \mathrm{FF}_{-}$CFB $\mathrm{i}_{\mathrm{i}, \mathrm{t}}+\alpha_{7} \mathrm{FF}_{-} \mathrm{DFT} \mathrm{i}_{\mathrm{i}, \mathrm{t}}+\alpha_{8} \mathrm{LOG}_{-} \mathrm{AKHINC} \mathrm{i}_{\mathrm{i}, \mathrm{t}}+\alpha_{9} \mathrm{CFB} \_\mathrm{AC} \mathrm{C}_{\mathrm{i}, \mathrm{t}}+\alpha_{10} \mathrm{AMPR} \mathrm{AC}_{\mathrm{i}, \mathrm{t}}+\varepsilon_{\mathrm{i}, \mathrm{t}}$

Avec :

$\mathrm{Y}=0$ pour les entreprises ayant un ratio INC_AC inférieur à la moyenne

$\mathrm{Y}=1$ pour les entreprises ayant un ratio INC_AC supérieur à la moyenne

DFT_AC : Dettes financières totales/Actif comptable

DCT_AK : Dettes à court terme/Actif comptable

DLT_AK : Dettes à long terme/Actif comptable

DCT_DFT : Dettes à court terme/Dettes financières totales

DEX_AK : Dettes d'exploitation/Actif comptable

FF_AK : Frais financiers/Actif comptable

FF_CFB : Frais financiers/Cash-flow brut

FF_DFT : Frais financiers /Dettes financières totales

LOG_AKHINC : Log décimal (actif comptable hors immobilisations incorporelles)

CFB_AC : Cash-flow brut/Actif comptable

AMPR_AC : Amortissement et provision/Actif comptable

$\varepsilon:$ le terme d'erreur

\section{Résultats et discussions}

Dans cette section, nous présenterons les résultats des analyses d'une part, et d'autre part ces résultats seront confrontés à ceux obtenus dans d'autres contextes.

\subsection{Résultats de l'analyse descriptive univariée}

L'analyse univariée (Tableau $n^{\circ} 3$ ) met en évidence les principales caractéristiques financières des entreprises de l'échantillon d'étude. S'agissant du modèle 1, les statistiques descriptives montrent que les investissements immatériels sont en moyenne de 0,0306 (tableau $n^{\circ} 3$ ). Ce qui indique que la proportion des actifs incorporels par rapport au total actif. En conséquence, nous pouvons dire que les entreprises cotées à la BRVM investissent faiblement dans les immatériels. Par ailleurs, la dispersion totale par rapport à cette moyenne est de 0,0511. En dépit de la faiblesse des investissements immatériels, le Q de Tobin moyen de l'échantillon est de 2,30. Le Q de Tobin, étant supérieur à l'unité, est un signal de l'existence d'opportunités d'investissement rentables. D'ailleurs, sous l'hypothèse d'efficience du marché boursier, la valeur boursière d'une société est égale à la valeur actualisée de ses flux de profits futurs. De ce fait, un $\mathrm{Q}$ de Tobin supérieur à 1 dénote que le marché anticipe une profitabilité de l'investissement au-delà de son coût (Kergueris, 2002). En outre, l'analyse univariée révèle que la dispersion par rapport à cette moyenne est plus marquée dans la dimension intraindividuelle $(1,62)$ que dans la dimension interindividuelle $(1,42)$. 
Tableau n ${ }^{\circ}$ : Statistique univariée du modèle 1

\begin{tabular}{|c|c|c|c|c|c|c|}
\hline Variable & & Mean & Std, Dev, & Min & Max & Observations \\
\hline \multirow{3}{*}{ INC_AC } & overall & \multirow{3}{*}{0,0306} & 0,0511 & 0 & 0,3792 & $\mathrm{~N}=161$ \\
\hline & between & & 0,0400 & 0,0001 & 0,1234 & $\mathrm{n}=$ \\
\hline & within & & 0,0327 & $-0,0928$ & 0,3033 & $\mathrm{~T}=$ \\
\hline \multirow{3}{*}{ Q } & overall & \multirow{3}{*}{2,2995} & 2,1377 & 0,4363 & 14,6315 & $\mathrm{~N}=$ \\
\hline & between & & 1,6221 & 1,0155 & 8,4223 & $\mathrm{n}=$ \\
\hline & within & & 1,4272 & $-5,2632$ & 8,5087 & $\mathrm{~T}=$ \\
\hline \multirow{3}{*}{ LOG_AKHINC } & overall & \multirow{3}{*}{10,8209} & 0,5748 & 9,3512 & 12,1185 & $\mathrm{~N}=$ \\
\hline & between & & 0,5761 & 9,7536 & 11,9974 & $\mathrm{n}=$ \\
\hline & within & & 0,1046 & 10,4185 & 11,1175 & $\mathrm{~T}=$ \\
\hline
\end{tabular}

\section{Source : Auteur}

Concernant le modèle 2 , les statistiques descriptives (tableau ${ }^{\circ} 4$ ) montrent que la proportion moyenne des dettes financières dans le total actif $\left(\mathrm{DFT} \_\mathrm{AC}=0,1371\right)$ est supérieure à la proportion moyenne de dette de court terme (DCT_AC $=0,545$ ). D'ailleurs, ce choix de la structure financière est corroboré par la faiblesse du poids des frais financiers $(1,13 \%)$ qui ne représente que $13 \%$ des dettes financières totales. En plus, les entreprises de l'échantillon sont en moyenne de grande taille $(10,82)$.

Tableau ${ }^{\circ} 4$ : Statistique univariée du modèle 2

\begin{tabular}{|c|c|c|c|c|c|c|}
\hline Variable & & Moyenne & Ecart type & Minimum & Maximum & Observations \\
\hline \multirow{3}{*}{$\mathrm{y}$} & overall & \multirow{3}{*}{0,931677} & 0,2530865 & 0 & 1 & $\mathrm{~N}=161$ \\
\hline & between & & 0,1919994 & 0,1428571 & 1 & $\mathrm{n}=$ \\
\hline & within & & 0,1690309 & 0,0745342 & 1,78882 & $\mathrm{~T}=$ \\
\hline \multirow{3}{*}{ dft_ac } & overall & \multirow{3}{*}{0,1370331} & 0,1153844 & 0 & 0,5655617 & $\mathrm{~N}=$ \\
\hline & between & & 0,1022754 & 0,0226931 & 0,5125105 & $\mathrm{n}=$ \\
\hline & within & & 0,0569699 & $-0,1179687$ & 0,3944052 & $\mathrm{~T}=$ \\
\hline \multirow{3}{*}{ dct_ac } & overall & \multirow{3}{*}{0,5456509} & 0,2429042 & 0,1712079 & 1,330084 & $\mathrm{~N}=$ \\
\hline & between & & 0,2305317 & 0,2234506 & 1,080102 & $\mathrm{n}=$ \\
\hline & within & & 0,0886033 & 0,2750585 & 0,8219673 & $\mathrm{~T}=$ \\
\hline \multirow{3}{*}{ dct_dft } & overall & \multirow{3}{*}{9,480677} & 13,23405 & 0 & 92,22293 & $\mathrm{~N}=$ \\
\hline & between & & 9,633338 & 1,357314 & 40,40551 & $\mathrm{n}=$ \\
\hline & within & & 9,263843 & $-13,46106$ & 77,77718 & $\mathrm{~T}=$ \\
\hline \multirow{3}{*}{ dex_ac } & overall & \multirow{3}{*}{0,257626} & 0,2427614 & 0 & 0,947399 & $\mathrm{~N}=$ \\
\hline & between & & 0,2288317 & 0 & 0,8297914 & $\mathrm{n}=$ \\
\hline & within & & 0,0923731 & $-0,0558501$ & 0,6523622 & $\mathrm{~T}=$ \\
\hline \multirow{3}{*}{ ff_cfb } & overall & \multirow{3}{*}{0,0771903} & 0,6907638 & $-7,186798$ & 2,575182 & $\mathrm{~N}=$ \\
\hline & between & & 0,2507548 & $-0,8059148$ & 0,724132 & $\mathrm{n}=$ \\
\hline & within & & 0,6454723 & $-6,303692$ & 3,201155 & $\mathrm{~T}=$ \\
\hline \multirow{3}{*}{ ff_ac } & overall & \multirow{3}{*}{0,0113188} & 0,0132904 & 0 & 0,0888183 & $\mathrm{~N}=$ \\
\hline & between & & 0,0122482 & 0 & 0,0530992 & $\mathrm{n}=$ \\
\hline & within & & 0,0056782 & $-0,0072472$ & 0,0470379 & $\mathrm{~T}=$ \\
\hline \multirow{3}{*}{ ff_dft } & overall & \multirow{3}{*}{0,1374348} & 0,2385011 & 0 & 1,929692 & $\mathrm{~N}=$ \\
\hline & between & & 0,142566 & 0 & 0,4367455 & $\mathrm{n}=$ \\
\hline & within & & 0,1931836 & $-0,2347665$ & 1,696077 & $\mathrm{~T}=$ \\
\hline \multirow{3}{*}{ logakhinc } & overall & \multirow{3}{*}{10,82088} & 0,5748301 & 9,351217 & 12,11851 & $\mathrm{~N}=$ \\
\hline & between & & 0,5761412 & 9,753628 & 11,99736 & $\mathrm{n}=$ \\
\hline & within & & 0,1045882 & 10,41847 & 11,11747 & $\mathrm{~T}=$ \\
\hline \multirow{3}{*}{ cfb_ac } & overall & \multirow{3}{*}{0,1342403} & 0,1378135 & $-0,3186821$ & 0,8816434 & $\mathrm{~N}=$ \\
\hline & between & & 0,0970896 & $-0,0129246$ & 0,3877128 & $\mathrm{n}=$ \\
\hline & within & & 0,0995975 & $-0,1715171$ & 0,9189214 & $\mathrm{~T}=$ \\
\hline \multirow{3}{*}{ ampr_ac } & overall & \multirow{3}{*}{0,0547763} & 0,0408638 & 0 & 0,2227895 & $\mathrm{~N}=$ \\
\hline & between & & 0,0376843 & 0,0093476 & 0,1490018 & $\mathrm{n}=$ \\
\hline & within & & 0,0174067 & 0,0115416 & 0,1540913 & $\mathrm{~T}=$ \\
\hline
\end{tabular}




\subsection{Résultats de l'analyse descriptive bivariée}

L'analyse bivariée a mis en évidence une corrélation significative et positive entre le Q de Tobin et la taille. Toutefois, cette corrélation est faible. Ce constat est similaire avec la décision d'activation des investissements immatériels. Contrairement à la taille, une corrélation négative est observée entre la décision d'activation des immatériels et la proportion des dettes financières dans l'actif comptable. Mais, cette corrélation est également faible.

De façon générale, la matrice des corrélations (tableau $n^{\circ}{ }^{5}$ ) indique des coefficients très faibles entre les variables explicatives. De ce fait, les variables explicatives sont indépendantes. Chaque variable recèle des informations différentes de celles des autres variables explicatives (Loulou et Triki, 2008). Ce qui voudrait dire qu'il y a absence de multicolinéarité entre lesdites variables.

L'analyse bivariée a mis en évidence une corrélation significative et positive entre le Q de Tobin et la taille. Toutefois, cette corrélation est faible. Ce constat est similaire avec la décision d'activation des investissements immatériels. Contrairement à la taille, une corrélation négative est observée entre la décision d'activation des immatériels et la proportion des dettes financières dans l'actif comptable. Mais, cette corrélation est également faible.

De façon générale, la matrice des corrélations (tableau $\mathrm{n}^{\circ}{ }^{5}$ ) indique des coefficients très faibles entre les variables explicatives. De ce fait, les variables explicatives sont indépendantes. Chaque variable recèle des informations différentes de celles des autres variables explicatives (Loulou et Triki, 2008). Ce qui voudrait dire qu'il y a absence de multicolinéarité entre lesdites variables.

Tableau $n^{\circ} 5$ : Statistique bivariée

\begin{tabular}{|c|c|c|c|c|c|c|c|c|c|c|c|}
\hline & $\mathrm{y}$ & dft_ac & dct_ac & dct_dft & dex_ac & ff_cfb & ff_ac & ff_dft & logakh c & cfb_ac & ampr_ac \\
\hline $\mathrm{y}$ & 1 & & & & & & & & & & \\
\hline dft_ac & 0,0011 & 1 & & & & & & & & & \\
\hline det_ac & $\begin{array}{c}- \\
0,1799 * \\
\end{array}$ & $\begin{array}{c}- \\
0,2298 * \\
\end{array}$ & 1 & & & & & & & & \\
\hline dct_dft & 0,0177 & $\begin{array}{c}- \\
0,4111 *\end{array}$ & $0,4555^{*}$ & 1 & & & & & & & \\
\hline dex_ac & 0,0734 & $\stackrel{-}{0,3435^{*}}$ & $0,6040 *$ & $0,3850 *$ & 1 & & & & & & \\
\hline ff_cfb & 0,0046 & 0,0196 & 0,0448 & 0,0315 & 0,1351 & 1 & & & & & \\
\hline ff_ac & 0,0861 & $0,2969 *$ & 0,0335 & 0,1339 & 0,0838 & 0,0715 & 1 & & & & \\
\hline ff_dft & 0,1349 & $\stackrel{-}{0,1766 *}$ & $0,1548^{*}$ & $0,5590^{*}$ & 0,0449 & 0,045 & $0,4665^{*}$ & 1 & & & \\
\hline $\begin{array}{c}\text { logakhin } \\
\mathrm{c}\end{array}$ & $0,1681 *$ & 0,103 & 0,0557 & 0,131 & 0,0808 & 0,0048 & 0,1261 & 0,0521 & 1 & & \\
\hline cfb_ac & 0,004 & 0,0456 & $-0,3990 *$ & 0,1137 & $-0,4383^{*}$ & 0,0101 & 0,0599 & 0,1058 & 0,1506 & 1 & \\
\hline ampr_ac & $0,2261 *$ & 0,0756 & $-0,5361 *$ & $-0,3455^{*}$ & $-0,4739 *$ & 0,0425 & 0,0075 & 0,146 & $0,3152 *$ & $0,5201 *$ & 1 \\
\hline
\end{tabular}




\section{Résultats de l'analyse multivariée et discussion}

Après avoir justifié la structure en panel avec le test de présence d'effets individuels de Fisher, le test de Hausman effectué indique que, dans le cadre de notre étude, le modèle à effets fixes est approprié. Ainsi, les résultats de la régression linéaire sur le Q de Tobin par la méthode des moindres carrés ordinaires révèlent que le pouvoir explicatif du modèle $\left(\mathrm{R}^{2}\right)$ est de 6,9\%. En d'autres termes, $6,9 \%$ de la variabilité des investissements immatériels activés (INC_AC) est expliquée par la valorisation du capital immatériel par le marché financier $(\mathrm{Q})$ et la taille. Il va s'en dire qu'il existe d'autres facteurs déterminant l'activation des immatériels non pris en compte dans le modèle 1.

L'analyse économétrique (tableau $\left.n^{\circ} 6\right)$ indique une relation positive et non significative $(0.000026)$ entre le montant des investissements immatériels activés (INC_AC) et leur valorisation par le marché financier. Ce résultat suggère que l'activation des investissements immatériels ne semble pas affecter la valorisation boursière des entreprises sur le marché financier régional, conformément à l'hypothèse d'efficience des marchés financiers. En outre, l'activation des immatériels n'est pas un signal pertinent qui informe les investisseurs sur les opportunités de croissance future des entreprises sur le marché financier régional. De ce fait, les entreprises ayant recours à cette option comptable ne sont pas bien valorisées par le marché financier. Ceci semble infirmer notre première hypothèse. D'ailleurs, ce résultat est conforme à ceux de Thibierge $(1997,2001)$ dans le contexte français, de Loulou et Triki (2008) dans le contexte français, de Ossonon (2017) dans le contexte ouest-africain et contraire aux conclusions des travaux de Turki et al (2006) dans le contexte tunisien.

Par contre, le coefficient de la taille est significatif au seuil de $1 \%$ avec un signe négatif (LOGAKHINC $=-0,002003$ ). Autrement dit, la taille de l'entreprise et le montant des immatériels activés évoluent en sens contraire. Ce résultat suggère que sur le marché financier régional, les grandes entreprises investissent moins dans l'immatériel. Deux raisons pourraient justifier cette constatation. En premier lieu, les entreprises de grande taille ne perçoivent pas les investissements immatériels comme porteurs d'avantages économiques futurs pour l'entreprise. En deuxième lieu, la majorité des grandes entreprises cotées sur le marché financier régional sont filiales de sociétés de groupes internationaux. Alors qu'en général, les décisions stratégiques sont prises par les sociétés mères. Partant de ce fait, les entreprises sur le marché régional ne détiennent pas assez d'actifs stratégiques tels que les immatériels (frais de recherche et développement, logiciels, brevets...). 
Tableau n ${ }^{\circ} 6$ : Résultats de la régression linéaire sur le $Q$ de Tobin (Modèle 1 )

\begin{tabular}{|l|c|c|}
\hline & XTGLS & XTREG_FE_ROBUST \\
\hline $\mathrm{Q}_{\mathrm{it}}$ & 0.000026 & -0.000264 \\
\hline LOG_AKHINC & $(1.18)$ & $(-0.25)$ \\
\hline & $-0.002003^{* * *}$ & -0.082855 \\
\hline cons & $(-3.31)$ & $(-1.39)$ \\
\hline & $0.024319^{* * *}$ & 0.927753 \\
\hline$N$ & $(3.64)$ & $(1.44)$ \\
\hline$R^{2}$ & 161 & 161 \\
\hline
\end{tabular}

\section{Source : Auteur}

Seuil de significativité :* $p<0.10, * * p<0.05, * * * p<0.01$

Avec :

INC_AC : proportion des actifs incorporels dans le total actif de l'entreprise i à l'année t $\mathrm{Q}: \mathrm{Q}$ de Tobin de l'entreprise i à l'année $\mathrm{t}$

LOG_AKHINC : taille de l'entreprise i à l'année t

D'ailleurs, les résultats des estimations du modèle 2 (tableau 7) à partir de la méthode probit sur données de panel indiquent que le coefficient de l'endettement de long terme (DFT_AC $=1,779863$ ) est positif et significatif au seuil de 1\%. Précisément, le niveau des dettes à long terme (DFT_AC) influence positivement la décision d'activation de l'immatériel. En d'autres termes, cela signifierait que les entreprises qui s'endettent sur le long terme ont une propension à activer les investissements immatériels. Alors que le niveau d'endettement à court terme (DCT_AC) l'influence négativement. Cela dit, quels que soient les indicateurs d'endettement retenus, les sociétés qui activent de l'incorporel sont significativement plus endettées que celles qui passent l'incorporel en charges, que ce soit en termes d'endettement court terme (DCT_AC), ou total des dettes financières (DFT_AC).

Par ailleurs, les résultats d'estimation aboutissent à une relation négative entre l'activation des immatériels et le poids de l'endettement (les frais financiers). En effet, eu égard les debt Covenants imposés par les banquiers, les entreprises ont tendance à optimiser leurs résultats comptables par l'activation des investissements immatériels en vue de se libérer des contraintes financières imposées par les contrats d'endettement. L'activation des investissements immatériels est appréhendée comme un subterfuge usité par les dirigeants des entreprises pour informer les créanciers sur la qualité de leurs projets d'investissement dans le but d'atténuer les coûts d'endettement. Ces résultats sont conformes aux prescriptions de la théorie de l'agence, de la théorie positive de la comptabilité, aux travaux de Daley et Vigeland (1983) dans le contexte américain, de Raffournier (1990), de Thibierge (2001) dans le contexte européen, Eierle et Wencki ((2016) dans le contexte allemand, de Turki et al (2006), de Chabchoub et Bouri (2011), dans le contexte tunisien ainsi que ceux de Koumagnanou (2018) dans le contexte togolais. Aussi, ces constatations plaident-elles en faveur de la validation de nos hypothèses $\mathrm{H}_{2 \mathrm{a}}$ et $\mathrm{H}_{2 \mathrm{~b}}$. Par conséquent, notre hypothèse $\mathrm{H}_{2}$ est validée. 
L'analyse économétrique du modèle de régression logistique dénote également un coefficient positif et significatif de la taille $(0,2364294)$ au seuil de $1 \%$. Ceci suggère que la probabilité d'activation augmente avec la taille des entreprises. Précisement, les grandes entreprises ont une propension à activer les investissements immatériels. En effet, les grandes entreprises ne capitalisent pas les investissements immatériels pour éviter les coûts politiques et réduire leur visibilité politique. Elles activent les immatériels pour informer le marché financier sur la qualité de leurs projets en vue d'une meilleure valorisation de leurs titres. Ce résultat contredit l'hypothèse des coûts politiques formulée dans la théorie positive de la comptabilité de Watts et Zimmerman (1986), les travaux de Daley et Vigeland (1983) dans le contexte américain, de Oswald (2000) dans le contexte britannique, de Landry et Callimaci (2003) dans le contexte canadien, de Turki et al (2006), de Triki et Loulou (2008) et Chachoub et Bouri (2011) dans le contexte tunisien. Toutefois, cette constatation est similaire aux travaux de Thibierge (1997) dans le contexte européen, de Saada (1995), de Ding et Stolowy (2003) et Cazavan-Jeny et Jeanjean (2005) dans le contexte français. De ce fait, notre hypothèse $\mathrm{H}_{3}$ est infirmée.

Tableau 7: Résultats de la régression logistique

\begin{tabular}{|l|c|c|c|}
\hline & XTPROBIT & PROBIT & $\begin{array}{c}\text { EFFETS } \\
\text { MARGINAUX }\end{array}$ \\
\hline dft_ac & $53.717516^{*}(1.75)$ & $53.721827^{* * *}(2.87)$ & $1,779863^{* * *}(3.33)$ \\
\hline dct_ac & $-35.407270(-1.59)$ & $-35.411127^{* *}(-2.57)$ & $-1,17321^{* *}(-2.73)$ \\
\hline dct_dft & $1.221367^{*}(1.65)$ & $1.221468^{* * *}(2.74)$ & $0,0404686^{* * *}(2.95)$ \\
\hline dex_ac & $19.206497^{*}(1.75)$ & $19.208151^{* * *}(2.78)$ & $0,6363872^{* * *}(2.93)$ \\
\hline ff_cfb & $3.047731(0.37)$ & $3.047471(1.42)$ & $0,1009661(1.27)$ \\
\hline ff_ac & $-1183.601027^{*}(-$ & $-1183.753084^{* * *}$ & $-39,21905^{* * *}(-3.39)$ \\
& $1.86)$ & $(-3.06)$ & \\
\hline ff_dft & $333.647603^{*}(1.74)$ & $333.699104^{* * *}(2.84$ & $11,05582^{* * *}(3.08)$ \\
\hline logakhinc & $7.135414(1.60)$ & $7.136176^{* * *}(2.50)$ & $0,2364294^{* * *}(2.75)$ \\
\hline cfb_ac & $4.513791(1.01)$ & $4.513368^{* *}(1.98)$ & $0,1495328^{* *}(2.03)$ \\
\hline ampr_ac & $75.343224^{* *}(2.26)$ & $75.344455^{* * *}(3,28)$ & $2,496245^{* * *}(6,25)$ \\
\hline cons & $-74.618591^{*}(-1.67)$ & $-74.625691^{* * *}(-$ & \\
& & $2.64)$ & \\
\hline /lnsig2u & $-13.803618(-0.02)$ & & 161 \\
\hline$N$ & 161 & 161 & \\
\hline Pseudo $R^{2}$ & & 0,7603 & \\
\hline Wald chi2(10) & 7.56 & 25.39 & \\
\hline Prob > chi2 & 0.6713 & 0.0046 & \\
\hline Log likelihood & -9.6184322 & -9.6184304 & \\
\hline
\end{tabular}

\section{Source : Auteur}

Avec :

$\mathrm{Y}=0$ pour les entreprises ayant un ratio INC_AC inférieur à la moyenne $\mathrm{Y}=1$ pour les entreprises ayant un ratio INC_AC supérieur à la moyenne 
DFT_AC : Dettes financières totales/Actif comptable

DCT_AK : Dettes à court terme/Actif comptable

DLT_AK : Dettes à long terme/Actif comptable

DCT_DFT : Dettes à court terme/Dettes financières totales

DEX_AK : Dettes d'exploitation/Actif comptable

FF_AK : Frais financiers/Actif comptable

FF_CFB : Frais financiers/Cash-flow brut

FF_DFT : Frais financiers /Dettes financières totales

LOG_AKHINC : Log décimal (actif comptable hors immobilisations incorporelles)

CFB_AC : Cash-flow brut/Actif comptable

AMPR_AC : Amortissement et provision/Actif comptable

$\varepsilon:$ le terme d'erreur

\section{Conclusion}

Les investissements immatériels sont devenus les catalyseurs de la création de richesse par les entreprises dans la nouvelle économie. Cependant, il existe un trou noir dans leur comptabilisation (Leymarie, 2001). En effet, le Syscohada, inspiré du modèle comptable euro-continental, offre un traitement alternatif inhérent à la comptabilisation des investissements immatériels. Par conséquent, l'objectif de cette recherche était d'analyser les facteurs de contingence de la comptabilisation des investissements immatériels.

A partir d'un échantillon de 23 entreprises non financières cotées sur la Bourse Régionales des Valeurs Mobilières d'Abidjan, sur la base d'une régression linéaire et d'une régression logistique, nos résultats prouvent que, sur le marché financier régional, les entreprises activent les investissements immatériels pour se libérer des contraintes financières imposées par les contrats d'endettement. D'ailleurs, les grandes entreprises sont enclines à activer les immatériels afin d'informer le marché financier sur la qualité de leurs projets contrairement à l'hypothèse des coûts politiques émise par la théorie politicocontractuelle de Watts et Zimmerman (1986).

En revanche, en dépit des enseignements tirés de notre étude, celle-ci comporte des limites. Premièrement, au niveau de la sélection de l'échantillon, nous n'avons pas constitué l'échantillon à partir d'entreprises qui mentionnent $\mathrm{R} \& \mathrm{D}$ dans leur rapport annuel, comme le suggère Triki et Loulou (2008), afin de mieux expliquer la politique d'activation des dépenses de Recherche et Développement. Deuxièmement, l'hypothèse d'opportunisme managérial n'a pas été testée dans notre contexte en raison de l'indisponibilité des données relatives à la rémunération des dirigeants.

En fonction des limites évoquées, nous proposons ultérieurement aux chercheurs de considérer un échantillon d'entreprises non cotées pour une comparaison des politiques comptables des entreprises cotées et non cotées, et de tester l'hypothèse d'opportunisme managérial. 


\section{BIBLIOGRAPHIE}

[1] D. Aboody, B. Lev, "The value relevance of intangibles: the case of software capitalization", Journal of Accounting Research, Vol. 36, pp. 161-191, 1998.

[2] C. H. Alcouffe, Y. Louzzani, "Mesures de l'investissement immatériel: indicateurs de mesure et de performance ", Cahier de recherche du LIRHE, note n, vol. 381, p. 165-185, 2003.

[3] E. Amir, B. Lev, "Value relevance of non financial information: The wireless communications industry", Journal of Accounting and Economics, 22: 139-158, 1996.

[4] A. S. Bharadwaj, S. G. Bharadwaj \& B. R. Konsynski, " Information technology effects on firm performance as measured by Tobin's q", Management science, 45(7), 1008-1024, 1999.

[5] E. Bartov, G. M. Bodnar, "Alternative accounting methods, information asymmetry and liquidity: Theory and evidence ". Accounting review, 397-418, 1996.

[6] I. Bouden, "Identification des incorporels lors des regroupements d'entreprises : impact sur le marché financier français". Euro-mediterranean economics and finance review 1 (3) : 93-104, 2006.

[7] J-F. Casta, "Théorie positive de la comptabilité, coordonné par B. Colasse. Encyclopédie de comptabilité", contrôle de gestion et audit, Economica, Paris, p. 1393-1402, 2009.

[8] A. Cazavan-Jeny, T. Jeanjean, "Pertinence de l'inscription à l'actif des frais de R\&D: une étude empirique". Comptabilité-Contrôle-Audit, 11(1), 5-21, 2005.

[9] A. Chabchoub, A. Bouri, "Etude empirique des déterminants des choix de comptabilisation des investissements immatériels : cas des entreprises tunisiennes", Comptabilites, economie et sociéte, Montpellier, France, 2011.

[10] S. Charfi, "Analyse empirique des facteurs explicatifs de la décision d'activation des dépenses de recherche et développement : Cas des entreprises françaises". Acte du 27ème congrès francophone de comptabilité, Tunisie, 2006.

[11] K.H. Chung et S.W. Pruitt, “A simple approximation of Tobin's q”, Financial Management, Vol. 23, n 3, pp. 70-74, 1994.

[12] L. Daley, R. Vigeland, "The effect of debt covenants and plitical costs on the choice of accounting methods", Journal of Accounting and Economics, Vol. 5, PP 195-211, 1983.

[13] D. Dhaliwal, W. G. Heninger, "The investment opportunity set and capitalization versus expensig methods of accounting choice issue, Accounting and Finance", Vol. 39, PP 151-176, 1999.

[14] Y. Ding, H. Stolowy, "Les facteurs déterminants de la stratégie des groupes français en matière de communication sur leurs activités de recherche et de développement". Revue Finance, contrôle et stratégie $6(1): 39-62,2003$.

[15] M.L. Defond, J. Jiambalvo, "Debt convenant violation and manipulation of accruals", Journal of Accounting and Economics. 17: 145-176, 1994.

[16] S. Draief, "Structure financière, gestion des résultats et caractéristiques de la firme". Comptabilite, Controle, Audit Et Institution(S), Tunisie, 2006.

[17] B. Eierle, S. Wencki, "The determinants of capitalising development costs in private companies: evidence from Germany", Journal of Business Economics, 86(3), 259-300, 2016.

[18] J. Gonne, J.R. Feudjo, "Comportement des entreprises en matière d'investissements immatériels: état des lieux et déterminants en contexte camerounais", La Revue Gestion et Organisation, 9(1), 37-50, 2017.

[19] M. Jensen, W. Meckling, "Theory of the Firm: Managerial Behavior, Agency Costs and Ownership Structure", Journal of Financial Economics, vol. 3, October, p. 305-360, 1976.

[20] K. Koumagnanou, "Facteurs déterminants la comptabilisation des actifs immatériels par les dirigeants des entreprises de l'espace OHADA : cas du Togo". Transitions numériques et informations comptables, May, Nantes, France, 2018.

[21] S. Landry, A. Callimaci, "The caracteristics of canadian firms that capitalize R\&D", Papier de recherche 07-2002 du centre de recherche en gestion, Université du Québec à Montréal, Canada, 2003.

[22] S. Leymarie, "La prise en compte de l'investissement immatériel-cas d'expérimentation", Document de recherche, 2001.

[23] S. Loulou, M. Triki, "Déterminants et pertinence de l'activation des dépenses de recherche \& développement dans le contexte des entreprises françaises", La Comptabilité, Le Contrôle et L'audit entre Changement et Stabilité, France, 2008.

[24] A. Maaloul, D. Zéghal, "Analyse du contenu informatif de la divulgation des informations sur le capital immatériel: le cas des entreprises américaines", Management international/International Management/Gestiòn Internacional, 19(3), 201-216, 2015. 
[25] Y. Mard, S. Marsat, "Gestion des résultats comptables et structure de l'actionnariat: le cas français". Comptabilité-Contrôle-Audit, 18(3), 11-42, 2012.

[26] B. Martory, C. Pierrat, "La gestion de l'immatériel", Edition Nathan, Paris, 1996.

[27] Martory B. (1999), "L'évaluation des immatériels : un point de vue gestionnaire”, Working paper, Université Paris-sud, Pesor.

[28] E. Nils, J. Huegh-Krohn, K.H. Knivsfla, "Accounting for Intangible Assets in Scandinavia, the UK, the US, and by the IASC: Challenges and a Solution", The International Journal of Accounting, Vol. 35, $\mathrm{n}^{\circ}$. 2, pp. 243-265, 2000.

[29] B. Olivier, “L'économie immatérielle, Industries et marchés d'expériences”, Gallimard, 2010.

[30] A.C. Ossonon, “Analyse de la pertinence de l'information comptable en matière d'investissements immatériels : une étude empirique sur le marché financier de l'UEMOA”, Doctorat en Sciences de Gestion, Abidjan, Université Felix Houphouët Boigny de Cocody, 2017.

[31] D. R. Oswald, "The determinants and value relevance of the choice of accounting for research and development expenditures in the United Kingdom”, European Accounting Association Congress, 2000.

[32] R.A. Posner, "Theories of Economic Regulation", Bell Journal of Economics and Management Science, $\mathrm{n}^{\circ}$ 5, p. 335-358, 1974.

[33] B. Raffournier, "La théorie positive de la comptabilité: une revue de la littérature". Economies et sociétés (Paris), 16, 1990.

[34] S. A. Ross, "The determination of financial structure: the incentive-signalling approach". The bell journal of economics, 23-40, 1977.

[35] T. Saada, "Les déterminants des choix comptables : étude des pratiques françaises et comparaison francoaméricaine", Comptabilité Contrôle Audit, tome 1, Vol. 2, Septembre, PP 52-74, 1995.

[36] B. Saoussen, "Effet des investissements immatériels sur la performance d'exploitation future: une étude empirique auprès des entreprises tunisiennes", Doctorat en Sciences de gestion, Tunis, Université La Manouba, ISCAE, 2010.

[37] D. J. Skinner, "The investment opportunity set and accounting procedure choice", Journal of Accounting and Economics, Vol. 16, n $1-2-3$, PP 407-445, 1993.

[38] C. Thibierge, "Contribution à l'étude des déterminants de la comptabilisation des investissements immatériels", Thèse de doctorat en science de gestion, Université Paris-Dauphine, 1997.

[39] C. Thibierge, "Actifs immatériels, valorisation boursière et contrainte d'endettement: étude empirique sur les marchés français et espagnol”, Congrès international de l'Association Française de Finance, Namur, 2830juin2001, pp 28, Actes en ligne, 2001.

[40] H. Turki, A. Abdelmoula, A. Jarboui, "La Comptabilisation des Dépenses Immatérielles: Quels Déterminants Empiriques?" Comptabilite, Controle, Audit Et Institution(S), Tunisie, 2006.

[41] M.A. Verdier, "Les choix comptables des dirigeants lors des réductions d'effectifs : contribution à l'hypothèse des coûts politiques", Gestion et management, Université Toulouse 1 Capitole. Français, 2015.

[42] R.L. Watts, J. Zimmerman, "Toward a Positive Theory of the Determination of Accounting Standards", The Accounting Review 53(1): 112-134, 1978.

[43] R.L. Watts, J. Zimmerman, “ Positive accounting theory”, Prentice hall, Englewood Cliffs, 1986. 